\title{
A New Predictor of in-Stent Restenosis in Patients Undergoing Elective Percutaneous Coronary İntervention: Triglyceride Glucose İndex
}

\author{
Muhsin Kalyoncuoglu, Alev Arat Ozkan'1, Ayşem Kaya², Yasin Yüksel ${ }^{3}$, Nafi Dogann ${ }^{4}$, Aziz Tevfik Gurmen ${ }^{1}$ \\ Department of Cardiology, Haseki Training and Research Hospital, University of Health Science, Departments of ${ }^{1}$ Cardiology and ${ }^{2}$ Biochemistry, Istanbul \\ University-Cerrahpasa Cardiology Institute, ${ }^{3}$ Department of Cardiology, Istanbul Training and Research Hospital, University of Health Science, \\ Istanbul, Turkey, ${ }^{4}$ Department of Cardiology, Private Echomar Hospital, Zonguldak, Turkey \\ ORCID: \\ Muhsin Kalyoncuoglu: https://orcid.org/0000-0002-6552-0942 \\ Alev Arat Ozkan: https://orcid.org/0000-0001-8064-7231 \\ Ayşem Kaya: https://orcid.org/0000-0003-3137-821X \\ Yasin Yuksel: https://orcid.org/0000-0002-0450-5503
}

\section{Abstract}

Objective: This study aimed to investigate whether triglyceride glucose (TyG) index predicts the development of in-stent restenosis (ISR) in patients undergoing percutaneous coronary intervention (PCI) for de novo lesions. Materials and Methods: This was a prospective case controlled study and includes 124 patients with a mean age of $57 \pm 9.1$. Control coronary angiography was performed in all patients enrolled in the study 6-12 months after the PCI. Blood sample triglycerides (TGs) and blood glucose concentrations were collected after at least $8 \mathrm{~h}$ of fasting. The TyG index was calculated as follows: $\log$ (serum TGs $[\mathrm{mg} / \mathrm{dL}] \times$ plasma glucose $[\mathrm{mg} / \mathrm{dL}] / 2$ ). The study cohort was divided into two groups as those with and without restenosis. Angiographically, stent restenosis, defined as the presence of $\geq 50 \%$ diameter narrowing either within the stent or within $5 \mathrm{~mm}$ proximally or distally to the stent margin. Results: The median follow-up time was 7.17 months ( \pm 2.01$)$. Forty-eight patients $(38.7 \%)$ had restenosis. Patients with ISR had more diabetes $(P<0.01)$ and CAD $(P=0.03)$. These also had higher TyG index $(P=0.04)$ and less drug-eluting stent implantation $(P=0.04)$. Diabetes (odds ratio [OR]: $1.927, P=0.03)$, stent type $(\mathrm{OR}: 0.342$, $P=0.02)$, and TyG index (OR: 4.144, $P=0.01)$ significantly predicted ISR. The receiver operating characteristics curve analysis revealed a cutoff value of TyG index $>4.71$ predicts the development of ISR. Conclusion: A TyG above 4.71 was found a risk factor for ISR during 1-year follow-up period after index elective PCI.

Keywords: Glucose index, glucose, restenosis, triglycerides

\section{INTRODUCTION}

Despite the technical and medical breakthroughs, percutaneous coronary intervention (PCI) is compromised by postinterventional in-stent restenosis (ISR), and the results are still not satisfactory. ${ }^{[1-3]}$ Clinical characteristics such as diabetes and hypertension are one of the main factors associated with the development of ISR. ${ }^{[4]}$ Hence, identifying high-risk patients

Received: 14-03-2021 Revised: 13-04-2021 Accepted: 26-04-2021

Published Online: 05-07-2021

\begin{tabular}{|l|l|}
\hline \multicolumn{3}{c|}{ Access this article online } \\
\hline Quick Response Code: & Website: \\
& \\
http://www.ijcva.com
\end{tabular}

with modifiable clinical features may be helpful for physicians to improve clinical outcomes.

Insulin resistance (IR) is one of the cornerstones of metabolic syndrome and is not only associated with increased risk

Address for correspondence: Dr. Muhsin Kalyoncuoglu

Department of Cardiology, Haseki Training and Research Hospital, University of Health Science, Istanbul 34147, Turkey. E-mail: mkalyoncuoglu80@gmail.com

This is an open access journal, and articles are distributed under the terms of the Creative Commons Attribution-NonCommercial-ShareAlike 4.0 License, which allows others to remix, tweak, and build upon the work non-commercially, as long as appropriate credit is given and the new creations are licensed under the identical terms.

For reprints contact: WKHLRPMedknow_reprints@wolterskluwer.com

How to cite this article: Kalyoncuoglu M, Ozkan AA, Kaya A, Yüksel Y, Dogan N, Gurmen AT. A new predictor of in-stent restenosis in patients undergoing elective percutaneous coronary İntervention: Triglyceride glucose İndex. Int J Cardiovasc Acad 2021;7:50-4. 
of developing cardiovascular disease (CVD) but also is significantly associated with the development of ISR and poor cardiovascular outcome in patients with or without diabetes. ${ }^{[5,6]}$ Direct IR measurement methods such as hyperinsulinemic euglycemic glucose clamp, insulin suppression test, are invasive, costly, and complex procedures. Triglyceride glucose (TyG) index, a product of triglyceride (TG) and fasting glucose, is newly defined and easily measurable marker with high sensitivity specificity in predicting the insulin resistance and its associated metabolic abnormalities. ${ }^{[7,8]}$ There are literature data showing that TyG index is associated with the increased risk of atherosclerotic CVD and poor cardiovascular outcome. ${ }^{[8-10]}$ On the other hand, to the best of our knowledge, there are no studies in the literature evaluating association between the TyG index and the development of ISR in patients undergoing elective PCI. In our study, we aimed to investigate the predictive accuracy of the TyG index in determining the ISR development.

\section{Materials and Methods}

This was a prospective case-controlled study. From October 2006 to December 2007, 292 consecutive patients, 37-84 years of age, undergoing coronary angiography and who consented to participate in the study were screened. A detailed medical history including hypertension, diabetes mellitus, family history of CAD, dyslipidemia, smoking, and history of CAD were obtained. Patients with acute coronary syndrome who need immediate PCI $(n=32)$, patients with restenosis or stent thrombosis $(n=2)$, those with PCI failure $(n=4)$, those who underwent only balloon angioplasty $(n=30)$, patients who were referred to coronary artery by-pass surgery $(n=2)$, those with any missing data $(n=30)$, those withdrew their consent and refuse to undergo an control angiography $(n=52)$, severe illness (cancer, hepatic, or renal disease with glomerular filtration rate $<30 \mathrm{~mL} / 1.73 \mathrm{~m}^{2} / \mathrm{min}$, chronic infections, or inflammatory disease; $n=0$ ), were excluded [Figure 1]. Furthermore, 16 patients over the age of 75 were excluded because they had an increased risk of malnutrition, which could have a confounding effect on study results. Finally, 124 patients underwent elective/planned PCI and gave written informed consent, constituted the actual study population. The study was approved by the local ethics committee.

Blood samples were collected from the antecubital vein from each patient after at least $12 \mathrm{~h}$ of fasting before the index intervention. Plasma levels of fasting blood glucose (FBG), creatinine, total cholesterol, TG, high-density lipoprotein cholesterol, and low-density lipoprotein cholesterol were measured using an automated chemistry analyzer. The TyG index was calculated as follows: log (serum TG $[\mathrm{mg} / \mathrm{dL}] \times$ plasma glucose $[\mathrm{mg} / \mathrm{dL}] / 2$ ).

Coronary angiograms were analyzed by two experienced interventionalist blinded to clinical data. PCIs were performed by a team of experienced operators and size, length, and type of the stent (bare metal stent [BMS] or drug-eluting stent [DES]) used was left to the discretion of the operator. According to the type of stent used, control coronary angiogram was performed 6-12 months after the index intervention. Primary end point was angiographically ISR which was defined as the presence of $\geq 50 \%$ diameter narrowing either within the stent or within $5 \mathrm{~mm}$ proximally or distally to the stent margin. The study cohort was divided into two groups as those with and without restenosis.

\section{Ethical statement}

Ethical statement; Istanbul University Cerrahpasa Medical Faculty, Prt number: 16831.

\section{Statistical analysis}

Continuous variables were expressed as mean \pm standard deviation and percentage for categorical variables. Nonnormally distributed variables are given as medians. Continuous variables were compared among the groups of patients with independent Student's $t$-test for normally distributed data. Group comparisons of categorical variables were performed using the Chi-square or Fisher's exact test, as appropriate. Multiple logistic regression analysis was utilized to identify factors related to ISR. Only the variables with a $P<0.1$ in the univariate analysis were incorporated into the multivariate logistic regression analysis. In order to evaluate the additional ability of TyG and to determine the optimal cutoff value for TyG index in predicting the development of ISR, receiver operating characteristic (ROC) curve analysis was used. Values of $P<0.05$ were considered significant in all analyses. Statistical analysis was performed using Statistical Package for the Social Sciences (SPSS) for Windows version 24.0 (Chicago, Illinois, USA).

\section{RESULTS}

This prospective case-controlled study included 124 consecutive patients with a mean age of $57 \pm 9.1$ years who underwent elective PCI. During the median 7-month (interquartile range: 6-8 months) follow-up period, four patients with stable angina pectoris, and three patients with acute coronary syndrome had coronary angiographies earlier than scheduled, but the remaining 117 patients underwent the planned control coronary angiography. Of these 124 patients, 48 (38.7\%) had restenosis, of whom $42(35.9 \%)$ had BMS implantation and $6(4.8 \%)$ had DES implantation. TyG index was statistically higher in patients with ISR $(P=0.04)$. Detailed demographic, clinical, laboratory, angiographic parameters of two groups are represented in Tables 1 and 2 . In order to determine the independent predictors of ISR using variables that exhibited statistically significant relation in the univariate analyses, we performed two multivariable logistic regression analyses. Multivariant analysis revealed that diabetes (odds ratio [OR]: 1.927, $P=0.03$ ), stent type (OR: $0.342, P=0.02$ ), and TyG index (OR: 4.144, $P=0.01$ ) significantly predicted the development of ISR [Table 3]. ROC analysis found a cutoff value of $>4.71$ for TyG index with a $82 \%$ sensitivity and $39 \%$ specificity (area-under-the-curve: $0.61 ; 95 \%$ confidence interval: $0.51-0.71, P=0.03$ ) in predicting the ISR [Figure 2]. 


\begin{tabular}{|c|c|c|c|c|}
\hline Parameters & All patients $(n=124)$ & No-restenosis $(n=76)$ & Restenosis $(n=48)$ & $P$ \\
\hline Age & $57 \pm 9.1$ & $57.3 \pm 9.1$ & $56.6 \pm 8.9$ & 0.65 \\
\hline Male, $n(\%)$ & $94(77.4)$ & $57(75)$ & $39(81.3)$ & 0.42 \\
\hline Hypertension, $n(\%)$ & $74(59.7)$ & $47(61.8)$ & $27(56.3)$ & 0.54 \\
\hline Hyperlipidemia, $n(\%)$ & $56(45.2)$ & $34(44.7)$ & $22(45.8)$ & 0.91 \\
\hline Diabetes mellitus, $n(\%)$ & $37(29.8)$ & $16(21.1)$ & $21(43.8)$ & $<0.01$ \\
\hline Current smoker, $n(\%)$ & $80(64.5)$ & $49(64.5)$ & $31(64.6)$ & 0.99 \\
\hline Family history, $n(\%)$ & $47(37.9)$ & $31(40.8)$ & $16(33.3)$ & 0.41 \\
\hline CAD history, $n(\%)$ & $67(54)$ & $47(61.8)$ & $20(41.7)$ & 0.03 \\
\hline LVEF, $n(\%)$ & $51.4 \pm 9.8$ & $50.7 \pm 10$ & $52.6 \pm 9.5$ & 0.32 \\
\hline \multicolumn{5}{|l|}{ Admission diagnosis } \\
\hline Stable angina, $n(\%)$ & $47(37.9)$ & $27(35.5)$ & $20(41.7)$ & 0.15 \\
\hline Unstable angina $n(\%)$ & $48(38.7)$ & $26(34.2)$ & $22(45.8)$ & \\
\hline NSTEMI, $n(\%)$ & $9(7.3)$ & $7(9.2)$ & $2(4.2)$ & \\
\hline STEMI, $n(\%)$ & $20(16.1)$ & $16(21.1)$ & $4(8.3)$ & \\
\hline Thrombolytic therapy & $15(12.1)$ & $10(13.2)$ & $5(10.4)$ & \\
\hline \multicolumn{5}{|l|}{ Laboratory parameters } \\
\hline FBG, $\mathrm{mg} / \mathrm{dl}, \mathrm{IQR}$ & $104(93-122)$ & $102(91-121)$ & $110(99-131)$ & 0.24 \\
\hline GFR, $\mathrm{mL} / 1.73 \mathrm{~m}^{2} / \mathrm{min}$ & $86 \pm 21$ & $87 \pm 20$ & $85 \pm 22$ & 0.71 \\
\hline HDL-C, mg/dL & $43 \pm 8.4$ & $43 \pm 8.3$ & $42 \pm 8.5$ & 0.88 \\
\hline $\mathrm{LDL}, \mathrm{mg} / \mathrm{dL}$ & $113 \pm 35$ & $110 \pm 34$ & $119 \pm 35$ & 0.12 \\
\hline TyG index, IQR & $4.87(4.66-5.0)$ & $4.84(4.61-5.0)$ & $4.93(4.74-5.1)$ & 0.04 \\
\hline \multicolumn{5}{|l|}{ Medications after discharge } \\
\hline Acetylsalicylic acid, $n(\%)$ & $120(96.8)$ & $72(94.7)$ & $48(100)$ & 0.11 \\
\hline Clopidogrel, $n(\%)$ & $113(91.1)$ & $67(88.2)$ & $46(95.8)$ & 0.14 \\
\hline RAS blockers, $n(\%)$ & $95(76.6)$ & $59(77.6)$ & $36(75)$ & 0.74 \\
\hline Beta blockers, $n(\%)$ & $105(84.7)$ & $63(82.9)$ & $42(87.9)$ & 0.49 \\
\hline Statins, $n(\%)$ & $109(85.5)$ & $65(85.5)$ & $44(91.7)$ & 0.31 \\
\hline
\end{tabular}

Continuous variables were presented as mean \pm SD if normally distributed and medians IQRs if not normally distributed, while categorical variables were given as percentages. CAD: Coronary artery disease, LVEF: Left ventricular ejection fraction, NSTEMI: Non-ST elevated myocardial infarction, STEMI: ST elevated myocardial infarction, FBG: Fasting blood glucose, GFR: Glomerular filtration rate, HDL-C: High density lipoprotein cholesterol, LDL-C: Low density lipoprotein cholesterol, TyG: Triglyceride glucose, RAS: Renin-angiotensin system, IQRs: Interquartile ranges, SD: Standard deviation

Table 2: Interventional and angiographic factors and association with in-stent restenosis

\begin{tabular}{|c|c|c|c|c|}
\hline Parameters & All patients $(n=124)$ & No-restenosis $(n=76)$ & Restenosis $(n=48)$ & $P$ \\
\hline \multicolumn{5}{|l|}{ Stented vessel, $n(\%)$} \\
\hline LAD and side branches & $51(41.1)$ & $30(39.5)$ & $21(43.8)$ & 0.19 \\
\hline LCx and side branches & $26(21)$ & $18(23.7)$ & $8(16.7)$ & \\
\hline RCA and side branches & $42(33.9)$ & $23(30.3)$ & $19(39.6)$ & \\
\hline By-pass graft & $5(4)$ & $5(6.6)$ & 0 & \\
\hline \multicolumn{5}{|l|}{ Type of stent, $n(\%)$} \\
\hline Bare metal stent & $97(78.2)$ & $55(72.4)$ & $42(87.5)$ & 0.04 \\
\hline Drug-eluting stent & $27(21.8)$ & $21(27.6)$ & $6(12.5)$ & \\
\hline Reference vessel diameter, $\mathrm{mm}$ & & $3.1 \pm 0.38$ & $3.0 \pm 0.33$ & 0.29 \\
\hline Final vessel diameter, mm & & $3.46 \pm 0.45$ & $3.35 \pm 0.34$ & 0.12 \\
\hline Length of lesion, mm & & $20 \pm 6.0$ & $21.7 \pm 5.9$ & 0.14 \\
\hline
\end{tabular}

\section{DISCUSSION}

ISR is an important clinical problem and continues to limit the long-term success of the PCI. ISR is a maladaptive response to localized injury of the coronary artery wall induced by coronary intervention and consists of complex molecular and cellular mechanisms such as releasing of inflammatory and chemoattractant factors leading to migration and proliferation of vascular smooth muscle cells, neointimal hyperplasia, and vascular remodeling. ${ }^{[1]]}$

TyG index is newly defined marker of identifying IR with a high sensitivity and specificity. ${ }^{[7,8]}$ To date, a few studies about the relationship between TyG and CVD such as coronary 
Table 3: Factors that were found to be independently associated with in-stent restenosis in univariate and multivariate cox regression analysis model

\begin{tabular}{|c|c|c|c|c|}
\hline Variables & Univariate OR (95\% Cl) & $P$ & Multivariate OR $(95 \% \mathrm{CI})^{*}$ & $P$ \\
\hline Diabetes & $1.989(1.114-3.552)$ & 0.02 & $1.927(1.061-3.501)$ & 0.03 \\
\hline CAD & $0.579(0.324-1.035)$ & 0.06 & $0.619(0.344-1.115)$ & 0.11 \\
\hline Type of stent & $0.284(0.118-0.679)$ & $<0.01$ & $0.342(0.140-0.837)$ & 0.02 \\
\hline TyG index & $3.119(1.036-9.389)$ & 0.04 & $4.144(1.331-12.906)$ & 0.01 \\
\hline
\end{tabular}

*The variables with a $P<0.1$ in the univariate analysis were incorporated into the multivariate cox regression analysis by using backward LR method, CAD: Coronary artery disease, TyG: Triglyceride glucose, OR: Odds ratio, CI: Confidence interval

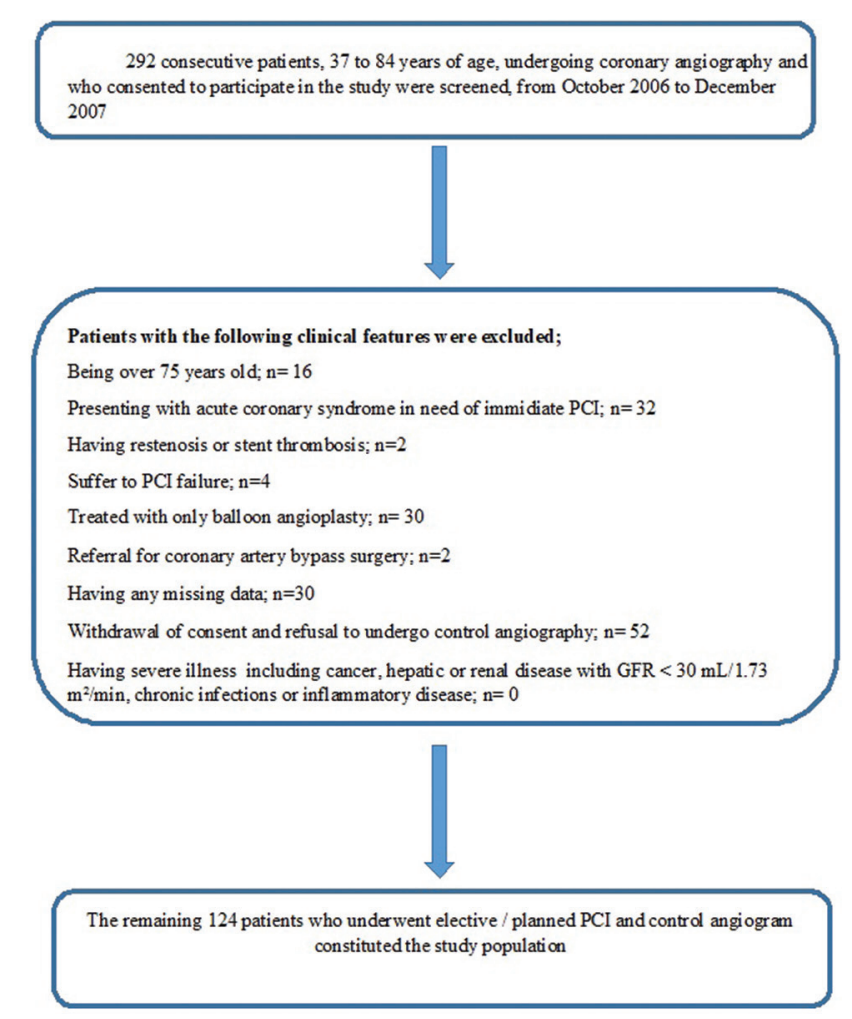

Figure 1: Study flow chart

artery calcification, peripheral artery disease severity, stroke, and carotid atherosclerosis have been addressed. ${ }^{[7-10,12,13]} \mathrm{In}$ addition, it was also found to be associated with increased CVD risk in apparently healthy individuals. ${ }^{[14]}$ In a study conducted by Jin et al., TyG index was significantly associated with future cardiovascular events and clinical outcomes in 3745 patients with stable CAD ${ }^{[9]}$ In another study, Mao et al. reported that the TyG index independently predicted the coronary artery disease severity and cardiovascular outcomes in 438 patients with no-ST elevated acute coronary syndrome. ${ }^{[8]}$ Although there are previous studies addressing the relationship between the TyG index and several CVD, there are no studies evaluating its relationship with the development of ISR in patients undergoing elective PCI. To the best of our knowledge, this is the first study to evaluate the predictive importance of the TyG index in ISR development. It is very difficult to reveal the exact mechanism underlying the relationship between the TyG index and ISR. The TyG index is a composite

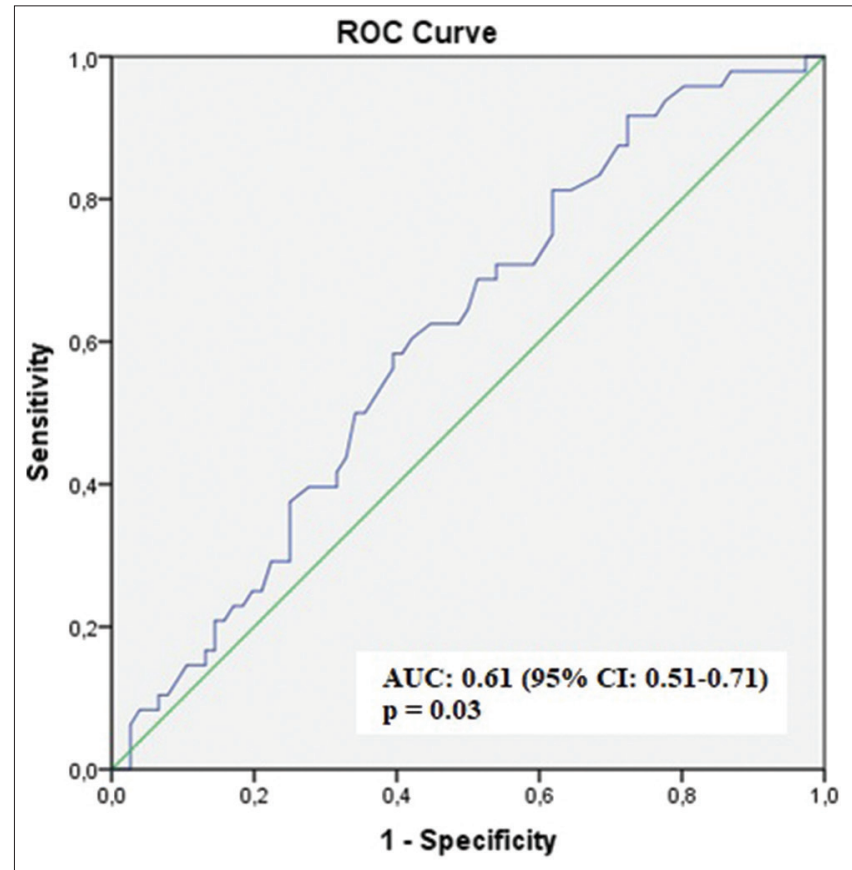

Figure 2: Receiver operating characteristic curve. The receiver operating characteristics curve analysis revealed a cutoff value of triglyceride glucose index $>4.71$ displayed $39 \%$ sensitivity and $82 \%$ specificity in predicting the in-stent restenosis. AUC: Area-under-the-curve, $\mathrm{Cl}$ : Confidence interval

indicator composed of TG and FBG and a marker of IR and metabolic syndrome. IR is characterized by an inappropriate physiologic response, in which insensitivity to insulin results in compensatory hyperinsulinemia. ${ }^{[15]}$ It has been suggested that hyperinsulinemia induces neointimal hyperplasia and promotes vascular smooth muscle cell proliferation which can be mediated by the convergent mechanisms of mitochondrial dysfunction and oxidative stress as well as activation of mitogen-activated protein kinase pathways. ${ }^{[5,6]}$ Therefore, both hyperglycemia and increased circulating levels of insulin may contribute to ISR. ${ }^{[16]}$ Indeed, several previous studies have also highlighted the relationship between IR and restenosis in patients undergoing PCI. ${ }^{[5,6]}$ Therefore, we think that high TyG index may be associated with ISR as an expression of increased IR.

Importantly, our study has some limitations. First, this study had a small sample size and relied on single-center experience. Second, ISR based on luminal stenosis was only evaluated by 
visual coronary angiograms and we did not include additional information about the quantitative assessment which was not as specific as intravascular ultrasound or optical coherence tomography. Third, we measured only baseline TyG index at admission and the changes that would be observed by serial measurements may have an additional predictive value. Fourth, we did not measure circulating insulin levels, hemoglobin A1c, homeostatic model assessment-IR, and IR index at the time of the index procedure. Although TyG was found to be a predictor of ISR development regardless of stent type, older generation DESs were used in the current study. Therefore, our findings need to be confirmed with the new generation DESs in larger prospective studies.

\section{Conclusion}

Our study showed that the TyG index independently predicted the development of ISR in patients undergoing elective PCI even after adjustment for stent type and diabetes. TyG may be a part of cardiovascular examination to identify individuals at high risk for ISR.

\section{Declaration of patient consent}

The authors certify that they have obtained all appropriate patient consent forms. In the form the patients have given their consent for their images and other clinical information to be reported in the journal. The patients understand that their names and initials will not be published and due efforts will be made to conceal their identity, but anonymity cannot be guaranteed.

\section{Financial support and sponsorship}

Nil.

\section{Conflicts of interest}

There are no conflicts of interest.

\section{RefERENCES}

1. Stettler C, Wandel S, Allemann S, Kastrati A, Morice MC, Schömig A, et al. Outcomes associated with drug-eluting and bare-metal stents: A collaborative network meta-analysis. Lancet 2007;370:937-48.

2. Pleva L, Kukla P, Hlinomaz O. Treatment of coronary in-stent restenosis: A systematic review. J Geriatr Cardiol 2018;15:173-84.
3. Giacoppo D, Alfonso F, Xu B, Claessen BE, Adriaenssens T, Jensen C, et al. Drug-coated balloon angioplasty versus drug-eluting stent 1mplantation in patients with coronary stent restenosis. J Am Coll Cardiol 2020;75:2664-78.

4. Wihanda D, Alwi I, Yamin M, Shatri H, Mudjaddid E. Factors associated with in-stent restenosis in patients following percutaneous coronary intervention. Acta Med Indones 2015;47:209-15.

5. Zhao LP, Xu WT, Wang L, Li H, Shao CL, Gu HB, et al. Influence of insulin resistance on in-stent restenosis in patients undergoing coronary drug-eluting stent implantation after long-term angiographic follow-up. Coron Artery Dis 2015;26:5-10.

6. Armstrong EJ, McCabe JM. Insulin resistance and in-stent restenosis: Could modulating insulin improve outcomes of percutaneous coronary intervention? Coron Artery Dis 2015;26:1-2.

7. Angoorani $\mathrm{P}$, Heshmat R, Ejtahed HS, Motlagh ME, Ziaodini H, Taheri $\mathrm{M}$, et al. Validity of triglyceride-glucose index as an indicator for metabolic syndrome in children and adolescents: The CASPIAN-V study. Eat Weight Disord 2018;23:877-83.

8. Mao Q, Zhou D, Li Y, Wang Y, Xu SC, Zhao XH. The triglyceride-glucose index predicts coronary artery disease severity and cardiovascular outcomes in patients with non-ST-segment elevation acute coronary syndrome. Dis Markers 2019;2019:1-11. Article ID: 6891537. doi: $10.1155 / 2019 / 6891537$.

9. Jin JL, Cao YX, Wu LG, You XD, Guo YL, Wu NQ, et al. Triglyceride glucose index for predicting cardiovascular outcomes in patients with coronary artery disease. J Thorac Dis 2018;10:6137-46.

10. Duran Karaduman B, Ayhan H, Keleș T, Bozkurt E. The triglyceride-glucose index predicts peripheral artery disease complexity Turk J Med Sci 2020;50:1217-22.

11. Kibos A, Campeanu A, Tintoiu I. Pathophysiology of coronary artery in-stent restenosis. Acute Card Care 2007;9:111-9.

12. Sánchez-Iñigo L, Navarro-González D, Fernández-Montero A, Pastrana-Delgado J, Martínez JA. Risk of incident ischemic stroke according to the metabolic health and obesity states in the Vascular-Metabolic CUN cohort. Int J Stroke 2017;12:187-91.

13. Irace C, Carallo C, Scavelli FB, De Franceschi MS, Esposito T, Tripolino C, et al. Markers of insulin resistance and carotid atherosclerosis. A comparison of the homeostasis model assessment and triglyceride glucose index. Int J Clin Pract 2013;67:665-72.

14. Sánchez-Íñigo L, Navarro-González D, Fernández-Montero A, Pastrana-Delgado J, Martínez JA. The TyG index may predict the development of cardiovascular events. Eur J Clin Invest 2016;46:189-97.

15. Cerf ME. Beta cell dysfunction and insulin resistance. Front Endocrinol (Lausanne) 2013;4:37.

16. Spadaccio C, Patti G, De Marco F, Coccia R, Di Domenico F, Pollari F, et al. Usefulness of preprocedural levels of advanced glycation end products to predict restenosis in patients with controlled diabetes mellitus undergoing drug-eluting stent implantation for stable angina pectoris (from the Prospective ARMYDA-AGEs Study). Am J Cardiol 2013;112:21-6. 